\title{
A novel, non-functional, COL1A1 polymorphism is not associated with lumbar disk disease in young male Greek subjects unlike that of the Sp1 site
}

\author{
Thalia Bei $^{2 *}$, Constantinos Tilkeridis ${ }^{1 *}$, Stavros Garantziotis ${ }^{3}$, \\ Sosipatros A. Boikos ${ }^{2}$, Konstantinos Kazakos ${ }^{1}$, Constantinos Simopoulos ${ }^{1}$, \\ Constantine A. Stratakis ${ }^{2}$
}

1 "Democritus" University of Thrace, Alexandroupolis, Greece, ${ }^{2}$ Section on Genetics and Endocrinology, Program on Developmental Endocrinology \& Genetics, National Institute of Child Health and Human Development, National Institutes of Health, Bethesda, Maryland, USA, ${ }^{3}$ Center for Recruitment of Military Personnel for Health Services (KEYG), Hellenic Armed Forces, Arta, Greece,

*Drs. Tilkeridis and Bei have contributed equally to this work and are thus sharing first authorship

\begin{abstract}
OBJECTIVE: We recently reported the association of the Sp1 site polymorphism of the COL1AI gene with lumbar disk disease (LDD). In the present study we searched for a different polymorphism of the COL1A1 gene (which is usually not in linkage disequilibrium with the Sp1 site) in subjects with LDD. DESIGN: Blood was collected from 24 Greek army recruits, aged $29 \pm 7.6$ years, with LDD, and 66 healthy men, aged $26 \pm 4.38$ years, matched for body mass index (BMI) and age, with normal BMD and with no history of trauma or fractures, who served as controls. DNA was extracted and the $C O L 1 A 1$ gene was sequenced. Of the control subjects, 12 were army recruits and 54 were selected from the general population. RESULTS: The four base-pair insertion polymorphism in the $C O L 1 A 1$ gene analyzed by polymerase chain reaction amplification of DNA produces two different fragments (alleles A1 and A2): 14 patients (58.3\%) were homozygous for $\mathrm{A} 2 \mathrm{~A} 2$, versus 35 controls (53\%), while 3 patients $(12.5 \%)$ were $\mathrm{A1A1}$, and 8 of the control subjects $(12 \%)$ had this genotype. There were no statistically significant differences in the presence of the two alleles of this polymorphism between patients with LDD and control subjects. CONCLUSIONS: A four base-pair insertion polymorphism of the COL1AI gene is not associated with the presence of LDD in young males, unlike the Sp1 site polymorphism of the same gene. These data reinforce the association between LDD and the functional polymorphisms of the Sp1 site by showing that other polymorphic sites of the of the COL1A1 gene in the same population of patients are not linked to the disease.
\end{abstract}

Key words: Collagen, $C O L 1 A 1$ polymorphism, Lumbar Disk Disease 


\section{INTRODUCTION}

Lumbar disk disease (LDD) belongs to the most common musculoskeletal disorders in the western world. It is one of the most frequent conditions for which patients seek medical care and a major cause of work disability. ${ }^{1,2}$ Although the factors leading to the development of LDD are multiple and diverse, there is significant evidence of a genetic predisposition. LDD has recently been associated with various polymorphisms of the collagen IX (COL9A3) gene. ${ }^{3}$ Collagen is the most abundant structural component of the extracellular matrix of connective tissues such as bone, cartilage, tendon, and skin. The collagen molecules are organized into rope-like fibrils, called heterotypic, because they are composed of multiple collagen types. All of these molecules have a triplehelical structure and feature uninterrupted repeats of the sequence Gly-X-Y (Gly is glycine, $\mathrm{X}$ is often proline, and $\mathrm{Y}$ is often hydroxyproline). Type I collagen is the major protein in skin, ligaments, and bone. It consists of 2 alpha- 1 and one alpha- 2 chains, which are encoded by the COL1A1 and COL1A2 genes, respectively. Mutations in the COL1A1 gene have been implicated in the pathogenesis of osteogenesis imperfecta, Ehlers-Danlos syndrome, dermatofibrosarcoma protuberans, and other diseases. ${ }^{4,5}$

Although both COL1A1 and COL1A2 are present in the intervertebral disk (in the annulus fibrosus primarily and the nucleus pulposus secondarily), ${ }^{4}$ there have been few studies of the COL1A1 gene in spine disease and these only in elderly individuals. ${ }^{5}$ Recent data strengthen the genetic component of LDD and link this disease to collagen-related genes. ${ }^{6,7}$ We recently reported in young male recruits of the Greek Army presenting with LDD an association of a functional polymorphism at the $\mathrm{Sp} 1$ site of the promoter region of the COL1A1 gene with LDD. ${ }^{8}$ Other genetic polymorphisms of the COL1A1 gene have not been studied.

A four base-pair insertion polymorphism in the 3' untranslated region (3'UTR) of the COL1A1 was recently reported: it is highly polymorphic producing a fragment of 430bp (allele A1) or a fragment of 434bp (allele A2) by simple DNA amplification. ${ }^{9}$ We investigated this COL1A1 polymorphism in the same population of patients that we had previously reported ${ }^{8}$ but with an enlarged group of controls to increase statistical power. Interestingly, the data show no association between this genetic change and LDD, further strengthening the notion that the Sp1 site's association with LDD is specific and not in linkage disequilibrium with a nearby locus or with other sequences in the large COL1A1 gene.

\section{METHODS}

\section{Subjects \& Diagnosis}

We collected blood from 24 Greek army recruits at the time of recruitment (during basic training) with LDD (mean age 29 \pm 7.6 years), and 66 healthy men, aged $26 \pm 4.38$ years, matched for body mass index (BMI), all with normal BMD and no history of trauma or fractures, who served as controls. ${ }^{8}$ Twelve of the controls were army recruits and 54 were from the general population. The only health complaint of the patient group was lower back pain. Military service is mandatory for all men in Greece and the draft is performed every few months. The young soldiers were part of a single draft group; they were evaluated at the time of their presentation to a military medical training site and were diagnosed with early LDD based on symptoms and imaging studies.

LDD was defined as the presence of unilateral radicular neurological symptoms involving the lower lumbar or sacral roots, such as muscular weakness of the appropriate index muscles, pain, or sensory deficits radiating below the knee and involving the appropriate dermatomes. Patients were excluded if they had a history of lower back trauma, a history of previous surgery to the affected area, or if they were older than 45 years. The neurological examination was performed in all patients by one of two investigators (CT, SG), who were unaware of the radiological findings. All patients had radiological confirmation of their disease; the visualized prolapse had to be compatible with the clinical symptoms. The radiographic studies were performed independently of this study as part of the medical evaluation of army recruits and consisted of MRI evaluation of the affected area. They were read by attending radiologists, who were unaffiliated to the investigators and blinded to the DNA and clinical findings.

The protocol was approved by the Department of 
Defense Health Service Review Committee, Athens, Greece. A control group of 12 Greek army recruits (mean age 24 years), without known history of LDD or complaints of lower back pain, was also studied. All subjects signed an informed consent form permitting the blood sampling. All patients and all controls belonged to the same draft group. An additional group of 54 gender- (males) and age-matched control subjects who, however, were not army recruits but selected from the general population, were genotyped to increase statistical strength to a total of 66 controls. Blood was collected in standard EDTA-coated tubes.

\section{DNA studies}

Genomic DNA was extracted from the blood samples by standard methods. To detect the A1 and A2 alleles, we used a polymerase chain reactionbased method; 4 positive samples were sequenced for confirmation of the sequence change. Primers used to amplify the 4bp insertion polymorphism were: 5'CCTTTCTGCTCCTTTCTCCA-3' (forward primer) and 5'-GCAACACAGTTACACAAGG-3' (reverse primer). The reverse primer was end-labelled with $\gamma-{ }^{32} \mathrm{P}$ using polynucleotide kinase (New England Biolabs, Beverly, MA). A fragment of 430bp (allele A1) and/or 434bp (allele A2) was obtained using a 6\% acrylamide gel (Promega, Madison, WI) according to a previously described protocol. . $^{8,10}$

\section{Statistics}

Results are expressed as percentages and $\chi^{2}$ analysis was instituted (with Fisher's correction, where appropriate): the distribution of the $\mathrm{A} 1$ and $\mathrm{A} 2$ alleles (A1A1, A1A2, and A2A2) was compared in the two groups (patients with LDD and controls). The data were also tested against the Hardy-Weinberg equilibrium by $\chi^{2}$ analysis. $P$-Values $<0.05$ were considered significant.

\section{RESULTS}

PCR analysis with end-labeling of the primer produced easily readable results (Figure 1). Sequencing confirmed the genotyping (data not shown).

Fourteen patients $(58.3 \%)$ were homozygous for $\mathrm{A} 2 \mathrm{~A} 2$ versus 35 controls (53\%), while 3 patients $(12.5 \%)$ were A1A1, and 8 of the control subjects $(12 \%)$ had this genotype (Table 1$)$. There were no

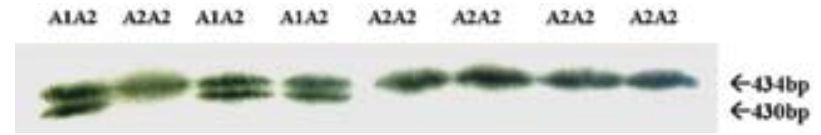

Figure 1. The A1 and A2 alleles of the 3'UTR of the COL1A1 gene are easily detectable by the method that was used in this study.

Table 1. Genotyping results for the 3'-UTR COL1A1 polymorphism

\begin{tabular}{lccc}
\hline Genotype & Controls $(\mathbf{n = 6 6})$ & Patients $(\mathbf{n = 2 4})$ & p value \\
\hline A1A1 & $8(12.1 \%)$ & $3(12.5 \%)$ & 1.000 \\
A1A2 & $23(34.8 \%)$ & $7(29.2 \%)$ & 0.801 \\
A2A2 & $35(53.0 \%)$ & $14(58.3 \%)$ & 0.811 \\
\hline
\end{tabular}

statistically significant differences in the presence of the two alleles of this polymorphism between patients with LDD and control subjects and there was no deviation from the Hardy-Weinberg equilibrium for both groups (patients and controls).

\section{DISCUSSION}

We recently reported an association of the $\mathrm{Sp} 1$ site COL1A1 polymorphism with $\mathrm{LDD}^{8}$ in the patients described in this report. The present study, although negative, strengthens our previous observations: the association of LDD with the functional Sp1 polymorphism of the COL1A1 gene is most likely specific and not in linkage disequilibrium with another locus, at least not as regards the 3' UTR site of the gene that was investigated in the present study.

Our previous report was, to our knowledge, the first of a positive association of a collagen I polymorphism with LDD. We demonstrated that patients with known LDD were much more likely to be homozygous for the $\mathrm{T} / \mathrm{T}$ Sp1 polymorphism than asymptomatic controls. Collagen I contributes a significant portion of the annulus fibrosus. ${ }^{4,5}$ The effect of the Sp1 polymorphism on collagen I appears to relate to an abnormal ratio of the collagen alpha- 1 chain production compared with alpha- 2 chain production; this may account for structural alterations, which in turn lead to a suboptimal annulus fibrosus quality, as is the case in bone. LDD may be a disease of more than the intervertebral disks alone. Although the literature 
on the subject has been conflicting, there is evidence that decreased BMD and intervertebral disk degeneration are positively correlated. ${ }^{3}$ It has been shown that the production of pro-collagen type I increases in LDD, possibly as a repair mechanism. ${ }^{4}$ Consistent with this hypothesis, a recent study suggested that collagen type-I in a glycosaminoglycan matrix induced proteoglycans synthesis by canine intervertebral disk cells. ${ }^{11}$ It is also interesting to note that in mice genetically engineered for reduced type I collagen, vertebral disk tissue was mechanically inferior when compared to that of control animals. ${ }^{12}$

There are some weaknesses in our study. First, the number of patients investigated is relatively small. Nevertheless, we believe that our patient group is representative. All our patients were army recruits with documented LDD. Some controls were army recruits as well. Since military service is mandatory for all Greek males, the patients presented here are a good cross-sectional sample for Greek males of the ages 18-40, which is the typical age range for draft in Greece. Second, not all control subjects were evaluated for radiological findings of LDD. It is known that a significant percentage of an asymptomatic population can have radiological findings of intervertebral disk degeneration. Our aim was to correlate radiographically proven, clinically relevant LDD with a known COL1A1 polymorphism and not to investigate the relation of radiological and genetic findings, as in other similar investigations. ${ }^{6,7}$

We report that a polymorphism of the COL1A1 gene not previously studied in LDD patients did not show an association with this condition in affected young soldiers, unlike the previously studied Sp1 sequence alleles. We conclude that the COL1A1 Sp1 site polymorphism appears to be specifically linked to LDD, although more polymorphic sequence changes of the COL1A1 locus need to be investigated to precisely define a region of association and the possible effect on disease expression.

\section{ACKNOWLEDGEMENTS}

We thank Dr. Evanggelos Kortessas, the Acting
Director of the KEYG Center at Arta, Greece, for his support of the study and approval of the research protocol.

\section{REFERENCES}

1. Anderson GB, 1999 Epidemiological features of chronic low-back pain. Lancet 354: 581-585.

2. Deen HG Jr, Yamodis ND, 1989 Lumbar disk disease in active duty military personnel. Mil Med 154: 502-504.

3. Paassilta P, Lohiniva J, Göring HH, et al, 2001 Identification of a novel common genetic risk factor for lumbar disk disease. JAMA 285: 1843-1849.

4. Antoniou J, Steffen T, Nelson F, et al, 1996 The human lumbar intervertebral disk. Evidence for changes in the biosynthesis and denaturation of the extracellular matrix with growth, maturation, ageing, and degeneration. J Clin Invest 98: 996-1003.

5. Pluijm SM, van Essen HW, Bravenboer N, et al, 2004 Collagen type I alpha1 Sp1 polymorphism, osteoporosis, and intervertebral disk degeneration in older men and women. Ann Rheum Dis 63: 71-77.

6. Seki S, Kawaguchi Y, Chiba K, et al, 2005 A functional SNP in CILP, encoding cartilage intermediate layer protein, is associated with susceptibility to lumbar disk disease. Nat Genet 37: 607-612.

7. Song YQ, Cheung KM, Ho DW, et al, 2008 Association of the asporin D14 allele with lumbar-disk degeneration in Asians. Am J Hum Genet 82: 744-777.

8. Tilkeridis C, Bei T, Garantziotis S, Stratakis CA, 2005 Association of a COL1A1 polymorphism with lumbar disk disease in young military recruits. J Med Genet 42: e44.

9. Nuytinck L, Coppin C, De Paepe A, 1998 A four base pair insertion polymorphism in the 3' untranslated region of the COL1A1 gene is highly informative for null allele testing in patients with osteogenesis imperfecta type I. Matrix Biology 16: 349-352.

10. Stratakis CA, Jenkins RB, Pras E, et al, 1996 Cytogenetic and microsatellite alterations in tumors from patients with the syndrome of myxomas, spotty skin pigmentation, and endocrine overactivity (Carney complex). J Clin Endocrinol Metab 81: 3607-3614.

11. Rong Y, Sugumaran G, Silbert JE, et al, 2002 Proteoglycans synthesized by canine intervertebral disk cells grown in a type I collagen-glycosaminoglycan matrix. Tissue Eng 8: 1037-1047.

12. Sarver JJ, Elliott DM, 2004 Altered disk mechanics in mice genetically engineered for reduced type I collagen. Spine 29: 1094-1098. 